\title{
Prevalence of resistance to aminoglycosides and fluoroquinolones among Pseudomonas aeruginosa strains in a University Hospital in Northeastern Poland
}

\author{
Anna Diana Michalska*, Pawel Tomasz Sacha*, Dominika Ojdana, Anna Wieczorek, \\ Elzbieta Tryniszewska
}

Department of Microbiological Diagnostics and Infectious Immunology, Medical University of Bialystok, Poland.

Submitted: Septermber 30, 2013; Approved: April 17, 2014.

\begin{abstract}
The present study was conducted to investigate the prevalence of genes encoding resistance to aminoglycosides and fluoroquinolones among twenty-five Pseudomonas aeruginosa isolated between 2002 and 2009. In PCR, following genes were detected: ant(2')-Ia in 9 (36.0\%), aac(6')-Ib in $7(28.0 \%), q n r B$ in $5(20.0 \%)$, $a p h(3 ")-I b$ in $2(8.0 \%)$ of isolates.
\end{abstract}

Key words: Pseudomonas aeruginosa, plasmid-mediated resistance to aminoglycosides and fluoroquinolones, aminoglycoside-modifying enzymes.

Pseudomonas aeruginosa is a non-fermentative, Gram-negative bacterium widespread in the natural and artificial environment. Characteristic feature of this pathogen is a remarkable ability to develop antimicrobial resistance, thus infections caused by multidrug-resistant (MDR) strains are associated with high mortality rate and elevated treatment cost (Lister et al., 2009). Many studies report that selection of highly resistant mutants occurs in Intensive Care Units and $P$. aeruginosa is a main cause of nosocomial infections (Wolska et al., 2012). Resistance to antibiotics may be linked both with chromosomal mutations and acquisition of resistance genes located on mobile genetic elements, such as plasmids, integrons, and transposons (Lister et al., 2009). From variety of plasmid-mediated aminoglycoside resistance mechanisms, the most commonly encountered is the production of aminoglycoside-modifying enzymes (Tada et al., 2013). High level of resistance to aminoglycosides can also be mediated with production of 16S rRNA methyltransferases, which preclude disturbance of protein synthesis caused by aminoglycoside molecule (Doi and Arakawa, 2007). Currently ten genes encoding these enzymes were detected, of which the most common are $\operatorname{arm} A$ and $r m t B$ (Deng et al., 2013). Plasmid-associated resistance to fluroquinolones can be mediated by the production of Qnr proteins, which preserve DNA gyrase and topoisomerase IV from inhibition by quinolones (Poirel, 2012). This mechanism contributes to low-level fluoroquinolone resistance, but it is able to broadening the mutant selecting window (Drlica and Zhao, 2007).

The aim of this study was to determine the prevalence of plasmid-mediated genes encoding aminoglycosidemodifying enzymes, 16S rRNA methyltransferases, and Qnr-like proteins among MDR P. aeruginosa strains.

Twenty-five nonduplicated $P$. aeruginosa strains were obtained from patients hospitalized in two Intensive Care Units at University Hospital of Bialystok (northeastern Poland) between July 2002 and October 2009. Isolates were selected due to their reduced susceptibility to aminoglycosides, fluoroquinolones, third- and fourth generation cephalosporins, and/or carbapenems. Identification and susceptibility testing were conducted using an automated VITEK 2 system with AST-N093 cards (bioMérieux, Marcy l'Etoile, France). Susceptibility to antibiotics was interpreted according to the EUCAST criteria published on February 11, 2013 (The European Committee on Antimicrobial Susceptibility Testing, 2013). The minimal inhibitory concentrations (MICs) of gentamicin, amikacin, netilmicin, ciprofloxacin, imipenem, meropenem, ceftazidime, and cefepime were determined by Etest technique (bioMérieux). Plasmid material was isolated from over-

Send correspondence to A.D. Michalska. Department of Microbiological Diagnostics and Infectious Immunology, Medical University of Bialystok, Waszyngtona Str. 15A, 15-269 Bialystok, Poland. E-mail: anna.michalska@umb.edu.pl.

*Both authors contributed equally to this work. 


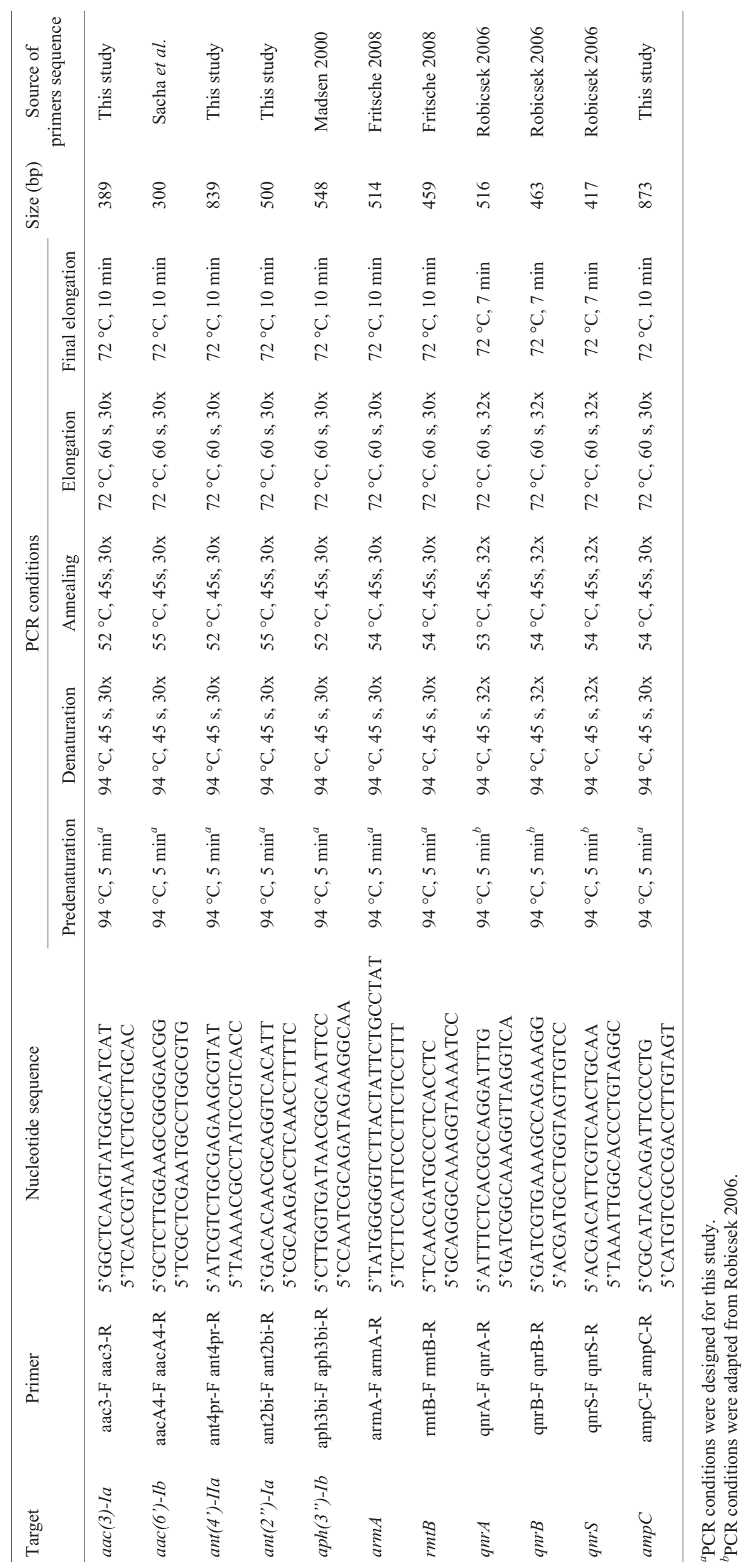


night cultures by Plasmid Mini Kit (A\&A Biotechnology, Gdynia, Poland). Screening of $a m p C$ gene was performed by polymerase chain reaction (PCR) with specific primer pair. Primers for amplification of $a a c\left(6^{\prime}\right)-I b, a a c(3)-I a$, $\operatorname{ant}\left(4^{\prime}\right)-I I a$, ant(2")-Ia, aph(3")-Ib, armA, rmtB, qnrA, $q n r B$, $q n r S$ genes were designed from sequences deposited in the National Center for Biotechnology Information website (http://www.ncbi.nlm.nih.gov/) or were selected from the literature (Table 1). Conditions of each PCR reaction are listed in Table 1. All PCR assays were performed in the LabCycler Gradient (SensoQuest GmbH, Goettingen, Germany). Sequencing of genes encoding aminoglycosidemodifying enzymes was conducted with primers listed in Table 1, using the 3500 Genetic Analyzer (Applied Biosystems, Foster City, USA).

The genes encoding aminoglycoside-modifying enzymes were identified in plasmid material of 13 strains (52.0\%). PCR assays revealed the presence of ant (2")-Ia gene in nine (36.0\%), aac (6')-Ib gene in seven $(28.0 \%)$, and $\operatorname{aph}(3$ ") $-\mathrm{Ib}$ gene in two (8.0\%) strains. Three isolates harbored two genes encoding aminoglycoside-modifying enzymes: $a a c\left(6^{\prime}\right)-I b$ and ant(2")-Ia in two strains; $\operatorname{ant}(2 ")-I a$ and $a p h(3 ")-I b$ in one strain. One isolate carried three genes for resistance to aminoglycosides: $a a c\left(6^{\prime}\right)-I b$, ant(2")-Ia, and aph(3")-Ib. QnrB gene related with plasmid-mediated resistance to quinolones was detected in five (20.0\%) strains. Sequencing of the PCR-positive products confirmed the presence of ant(2')-Ia, aac(6')-Ib, $a p h(3 ")-I b$, and qnrB1 genes in particular strains (GenBank accession numbers: ant(2")-Ia X04555.1; $a a c(6$ ')-Ib JF901756.1; aph(3")-Ib M28829.1, qnrB1 DQ777878.1). Genes aac(3)-Ia, ant(4')-IIa, armA, rmtB, $q n r A$, and $q n r S$ were not identified in plasmid DNA of tested strains. Characteristic of MDR strains with identified genes for resistance to aminoglycosides and quinolones are shown in Table 2. The highest efficiency among antimicrobials showed ceftazidime $(68.0 \%$ of all tested strains were susceptible). The only aminoglycoside active against tested strains was amikacin $(8.0 \%$ of all tested strains). Higher resistance rates were observed in strains carrying genes encoding aminoglycoside-modifying enzymes, than in strains without this genes detected. Level of resistance to ciprofloxacin was noticeably higher in strains harboring $q n r B$ gene than in strains without this gene identified $\left(\mathrm{MIC}_{50}: \geq 32 v s\right.$. MIC50: 8). As for carbapenems, more isolates were susceptible to imipenem $(28.0 \%)$ than meropenem $(24.0 \%)$.

Over the years, numerous studies reported the increasing prevalence of MDR $P$. aeruginosa in hospital environments all around the world. The present study focused on the investigation of plasmid-mediated resistance to aminoglycosides and fluoroquinolones in hospital located in northeastern Poland. The most frequently detected gene was $\operatorname{ant}(2$ ")-Ia (36.0\%). Spanish research also revealed that ant(2")-Ia gene occurs most often among

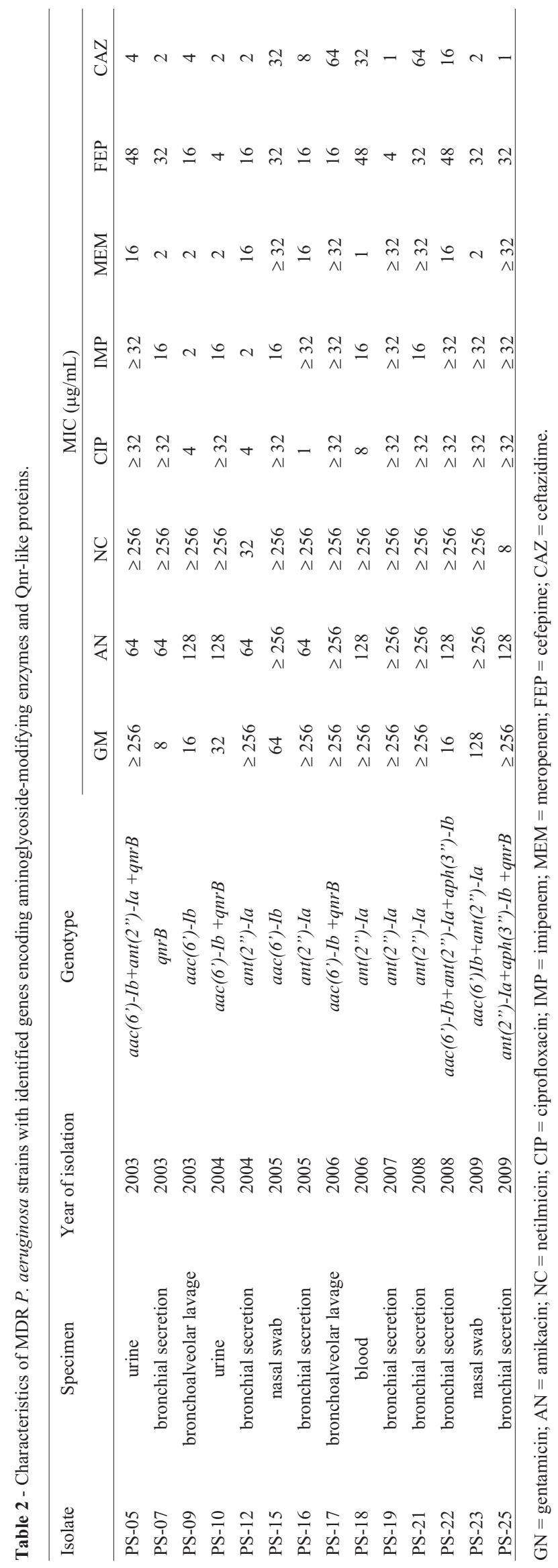


$P$. aeruginosa strains - it was identified in $65.0 \%$ (Fernandez et al., 2013), while in Iranian study it was observed in $28.0 \%$ of tested isolates (Vaziri et al., 2011). Our earlier investigation conducted on MDR $P$. aeruginosa reported the presence of $a a c\left(6^{\prime}\right)-I b$ gene in $58.3 \%$ of isolates (Sacha et al., 2012), whereas in this assay it was detected in $28.0 \%$ of tested strains. PCR study performed to detect genes involved in the production of Qnr-like proteins revealed the presence of $q n r B$ in $20.0 \%$ of tested strains. Among Enterobacteriaceae screened for production of plasmidmediated fluoroquinolone resistance determinants, $q n r B$ was reported as most prevalent gene (Kim et al., 2009). Earlier Polish study demonstrated that aminoglycoside and fluoroquinolone resistance rates were comparable to our results: amikacin $(91.0 \%$ vs. $92.0 \%)$, gentamicin $(98.0 \%$ vs. $100.0 \%)$, ciprofloxacin $(98.0 \%$ vs. $100.0 \%)$. Percentage of strains resistant to beta-lactams was even higher: $93 \%$ were resistant to ceftazidime, $89 \%$ to cefepime, $41 \%$ to imipenem, 88\% to meropenem (Paluchowska et al., 2012). Resistance rates of MDR isolates obtained from 10 Spanish hospitals were similar to those of our strains in the case of imipenem (66.67\% vs. 72\%), ceftazidime (40\% vs. 32\%), cefepime (73.33\% vs. 88\%) (Cabot et al., 2012).

This research focused on investigating the most commonly reported plasmid-mediated factors of aminoglycoside and fluoroquinolone resistance, and further assays are necessary to determine the other causes of antimicrobial resistance.

This study was partially supported by funds from Leading National Research Center in Bialystok (KNOW 50/2013).

\section{References}

Cabot G, Ocampo-Sosa AA, Domínguez MA, Gago JF, Juan C, Tubau F, Rodríguez C, Moyà B, Peña C, Martínez-Martínez L, Oliver A, Spanish Network for Research in Infectious Diseases (2012) Genetic markers of widespread extensively drug-resistant Pseudomonas aeruginosa high-risk clones. Antimicrob Agents Chemother 56:6349-6357.

Dallenne C, Da Costa A, Decré D, Favier C, Arlet G (2010) Development of a set of multiplex PCR assays for the detection of genes encoding important beta-lactamases in Enterobacteriaceae. J Antimicrob Chemother 65:490-495.

Deng YT, Zeng ZL, Tian W, Yang T, Liu J-H (2013) Prevalence and characteristics of $r m t B$ and qep $A$ in Escherichia coli isolated from diseased animals in China. Front Microbiol 4:198.

Doi Y, Arakawa Y (2007) 16S ribosomal RNA methylation: emerging resistance mechanism against aminoglycosides. Clin Infect Dis 45:88-94

Drlica K, Zhao X (2007) Mutant selection window hypothesis updated. Clin Infect Dis 44:681-688.

Fernández M, Ocampo-Sosa A, Cabot G, Tubau F, Peña C, Rodríguez C, Oliver A, Martínez-Martínez L, Spanish Network for Research in Infectious Diseases (2013) Prevalence of aminoglycoside-modifying enzyme genes in Pseudomonas aeruginosa isolated from bacteraemia in Spanish hospitals. 23rd European Congress of Clinical Microbiology and Infectious Diseases, Berlin.

Fritsche TR, Castanheira M, Miller GH, Jones RN, Armstrong ES (2008) Detection of methyltransferases conferring highlevel resistance to aminoglycosides in Enterobacteriaceae from Europe, North America, and Latin America. Antimicrob Agents Chemother 52:1843-1845.

Kim HB, Park CH, Kim CJ, Kim EC, Jacoby GA, Hooper DC (2009) Prevalence of plasmid-mediated quinolone resistance determinants over a 9-year period. Antimicrob Agents Chemother 53:639-645.

Lister PD, Wolter DJ, Hanson ND (2009) Antibacterial-resistant Pseudomonas aeruginosa: clinical impact and complex regulation of chromosomally encoded resistance mechanisms. Clin Microbiol Rev 22:582-610.

Madsen L, Aarestrup FM, Olsen JE (2000) Characterisation of streptomycin resistance determinants in Danish isolates of Salmonella Typhimurium. Vet Microbiol 75:73-78.

Paluchowska P, Skalkowska M, Spelak A, Budak A (2012) Occurrence of alert pathogens in hospital environment Part II. Multidrug-resistant non-fermenting bacilli. Med Dosw Mikrobiol 64:45-53.

Poirel L, Cattoir V, Nordmann P (2012) Plasmid-mediated quinolone resistance; Interactions between human, animal, and environmental ecologies. Front Microbiol 3:24

Robicsek A, Strahilevitz J, Sahm DF, Jacoby GA, Hooper DC (2006) qnr prevalence in ceftazidime-resistant Enterobacteriaceae isolates from the United States. Antimicrob Agents Chemother 50:2872-2874.

Sacha P, Jaworowska J, Ojdana D, Wieczorek P, Czaban S, Tryniszewska E (2012) Occurrence of the aacA4 gene among multidrug resistant strains of Pseudomonas aeruginosa isolated from bronchial secretions obtained from the Intensive Therapy Unit at University Hospital in Bialystok, Poland. Folia Histochem Cytobiol 50:322-324.

Tada T, Miyoshi-Akiyama T, Shimada K, Shimojima M, Kirikae T (2013) Novel 6'-n-aminoglycoside acetyltransferase AAC(6')-Iaj from a clinical isolate of Pseudomonas aeruginosa. Antimicrob Agents Chemother 57:96-100.

The European Committee on Antimicrobial Susceptibility Testing. Breakpoint tables for interpretation of MICs and zone diameters. Version 3.1, 2013. Available at: http://www.eucast.org/fileadmin/src/me-

dia/PDFs/EUCAST_files/Breakpoint_tables/Breakpoint table_v_3.1.pdf. Accessed 30 September 2013

Vaziri F, Peerayeh SN, Nejad QB, Farhadian A (2011) The prevalence of aminoglycoside-modifying enzyme genes (aac(6')-I, aac(6')-II, ant(2')-I, aph (3')-VI) in Pseudomonas aeruginosa. Clinics 66:1519-1522.

Wolska K, Kot B, Jakubczak A (2012) Phenotypic and genotypic diversity of Pseudomonas aeruginosa strains isolated from hospitals in Siedlce (Poland). Braz J Microbiol 43:274-282.

All the content of the journal, except where otherwise noted, is licensed under a Creative Commons License CC BY-NC. 